\title{
Genome-wide annotation and functional identification of aphid GLUT-like sugar transporters
}

\author{
Daniel RG Price* and John A Gatehouse
}

\begin{abstract}
Background: Phloem feeding insects, such as aphids, feed almost continuously on plant phloem sap, a liquid diet that contains high concentrations of sucrose (a disaccharide comprising of glucose and fructose). To access the available carbon, aphids hydrolyze sucrose in the gut lumen and transport its constituent monosaccharides, glucose and fructose. Although sugar transport plays a critical role in aphid nutrition, the molecular basis of sugar transport in aphids, and more generally across all insects, remains poorly characterized. Here, using the latest release of the pea aphid, Acyrthosiphon pisum, genome we provide an updated gene annotation and expression profile of putative sugar transporters. Finally, gut expressed sugar transporters are functionally expressed in yeast and screened for glucose and fructose transport activity.

Results: In this study, using a de novo approach, we identified 19 sugar porter (SP) family transporters in the A. pisum genome. Gene expression analysis, based on 214, 834 A. pisum expressed sequence tags, supports 17 sugar porter family transporters being actively expressed in adult female aphids. Further analysis, using quantitative PCR identifies 4 transporters, A. pisum sugar transporter 1, 3, 4 and 9 (ApST1, ApST3, ApST4 and ApST9) as highly expressed and/or enriched in gut tissue. When expressed in a Saccharomyces cerevisiae hexose transporter deletion mutant (strain EBY.WW4000), only ApST3 (previously characterized) and ApST4 (reported here) transport glucose and fructose resulting in functional rescue of the yeast mutant. Here we characterize ApST4, a 491 amino acid protein, with 12 predicted transmembrane regions, as a facilitative glucose/fructose transporter. Finally, phylogenetic reconstruction reveals that ApST4, and related, as yet uncharacterized insect transporters are phylogenetically closely related to human GLUT (SLC2A) class I facilitative glucose/fructose transporters.

Conclusions: The gut enhanced expression of ApST4, and the transport specificity of its product is consistent with ApST4 functioning as a gut glucose/fructose transporter. Here, we hypothesize that both ApST3 (reported previously) and ApST4 (reported here) function at the gut interface to import glucose and fructose from the gut lumen.
\end{abstract}

Keywords: Facilitative transport, Hexose sugars, Major facilitator superfamily, Membrane transport, Uniport

\section{Background}

Phloem feeding insects, which includes aphids, whiteflies, psyllids and planthoppers, feed solely on phloem sap, which contains high concentrations of sucrose (a disaccharide sugar comprising of glucose and fructose). Utilizing sucrose as its main respiratory substrate the pea aphid, Acyrthosiphon pisum, catabolizes 15 - 30\% of its ingested sucrose in oxidative pathways. A larger proportion, close to $50 \%$ of

\footnotetext{
* Correspondence: danrgp@gmail.com

School of Biological and Biomedical Sciences, Durham University, South

Road, Durham DH1 3LE, UK
}

\section{Biomed Central}

(c) 2014 Price and Gatehouse; licensee BioMed Central Ltd. This is an Open Access article distributed under the terms of the Creative Commons Attribution License (http://creativecommons.org/licenses/by/4.0), which permits unrestricted use,

distribution, and reproduction in any medium, provided the original work is properly credited. The Creative Commons Public Domain Dedication waiver (http://creativecommons.org/publicdomain/zero/1.0/) applies to the data made available in this article, unless otherwise stated.

the ingested sucrose, is incorporated into aphid tissues $[1,2]$. While sucrose is the main carbon source for $A$. pisum, sucrose itself is not transported across the gut epithelium [3]. Instead sucrose is hydrolyzed in the gut lumen by an $\alpha$-glucosidase to its constituent monosaccharides, glucose and fructose [4-6], and these hexose sugars are transported. Although sugar transport plays a critical role in aphid nutrition, the molecular basis of sugar transport in phloem feeding insects is poorly characterized. In this study, we address this knowledge 
gap by testing our prediction that $A$. pisum uses facilitative transporters belonging to the major facilitator superfamily for hexose sugar transport.

The major facilitator superfamily (MFS), one of the largest transporter superfamilies, currently contains 82 families, with each family specific for a group of compounds [7]. Within the MFS, sugars are transported by sugar porter family transporters [transporter classification number (T.C \#) 2.A.1.1]. The sugar porter family is essentially ubiquitous and found throughout the tree of life. Currently, our best insight into sugar porter function comes from detailed structure and function analyses of human transporters (reviewed in [8-11]). Human sugar porter family transporters (gene symbol SLC2A, protein symbol GLUT) contain 14 members, which are grouped according to their sequence similarity (GLUTs Class I - III). By far the best-described are GLUT class I transporters (GLUTs $1-4$ and 14) and GLUT class II transporters (GLUTs 5, 7, 9 and 11), which mediate equilibrative, energy independent membrane transport of hexose sugars down their concentration gradient. GLUT class III transporters (GLUTs 6, 8, 10, 12 and 13) are more recently discovered, as a result of the sequencing of the human genome, and their function is less well-described $[8,11]$. Predominant sugars transported by human GLUTs include glucose, galactose, fructose, and myoinositol [8-11].

Previously, using the initial release of the A. pisum genome sequence (assembly Acyr_1.0) [12] we identified an expanded family of major facilitator superfamily sugar transporters [13]. The most highly expressed sugar transporter (according to number of gut expressed sequence tags) was named $A$. pisum transporter 3 (ApST3, ACYPI004204). ApST3 is globally highly expressed in insect tissues and is enriched 2.2-fold in gut tissues, relative to whole insect expression levels [13]. Functionally, ApST3 is a low affinity fructose ( $K_{\mathrm{m}} 47 \mathrm{mM}$ fructose) and low affinity glucose $\left(K_{\mathrm{m}} 66 \mathrm{mM}\right.$ glucose $)$ facilitative transporter [13]. In a related study by Kikuta et al., 18 putative sugar transporters were identified in phloem feeding insect, the rice brown planthopper, Nilaparvata lugens. Of these, 6 transporters were highly expressed at the gut interface compared to whole insect levels [14]. The $N$. lugens ortholog of ApST3, designated $N$. lugens sugar transporter 6 (NIST6, BAI83420) has 43\% amino acid sequence identity and $60 \%$ sequence similarity to ApST3. Transporter NIST6 is enriched $\sim 9$-fold in gut tissues, relative to whole insect expression levels and has an uptake profile similar to ApST3, transporting both glucose and fructose [14]. Based on gene expression pattern, and substrate selectivity, it likely that $A$. pisum ApST3 and the $N$. lugens ortholog NIST6 import glucose and fructose at the gut interface and contribute to carbon nutrition in these phloem feeding insects.
Here, in an effort to more thoroughly describe the extent of $A$. pisum sugar transport at the gut interface we utilize a genome-wide approach to identify sugar transporters and screen for glucose and fructose transport function. In this study, using a de novo approach we identify 19 sugar porter family transporters in the latest release A. pisum genome (assembly Acyr_2.0, ABLF00000000.2). Gene expression analysis reveals that 4 of these sugar porter family transporters (ApST1, ApST3, ApST4 and ApST9) are highly expressed at the $A$. pisum gut interface. When functionally expressed in Saccharomyces cerevisiae hexose transport deletion mutant (strain EBY.VW4000) only ApST3 (previously described, [13]) and ApST4 (described here) functionally rescue the hexose transport mutant. We report here the detailed functional characterization of $A$. pisum sugar transporter 4. (ApST4, ACYPI0010980). ApST4 is a facilitative glucose and fructose transporter that is structurally, functionally and phylogenetically related to mammalian GLUT class I transporters [8-11]. ApST4 is globally highly expressed throughout the aphid, enriched 5.9-fold in gut tissues relative to whole insect expression levels, and transports glucose and fructose with high affinity.

\section{Results}

The A. pisum genome contains a large family of sugar porters

The latest release of the A. pisum genome (assembly Acyr_2.0) contains 19 sugar porter family transporters that match the TIGRFAMs sugar porter motif (TIGR00879). All gene annotations are presented in Additional file 1: Table S1. Gene expression analysis, based on 214, $834 \mathrm{~A}$. pisum expressed sequence tags (GenBank dbEST release 130101) supports 17 of these sugar porter family transporters being expressed in mixed population adult A. pisum. The two remaining transporters (ApST24 and ApST25) are not represented in the $A$. pisum expressed sequence tag (EST) dataset, suggesting that they are not expressed or only expressed at very low levels. One of the expressed transporters, ApST26, is only represented by male ESTs, suggesting that this transporter is male-specific.

The complement of sugar porter family transporters in other insect species included in our analysis (for species information see Methods) ranges between $8-28$. Comparatively, with 19 predicted sugar porter family transporters, A. pisum contains a large family of sugar porters. Only the red flour beetle, $T$. castaneum, with 28 sugar porter family transporters, encodes a larger number of transporters (Additional file 1: Table S2).

\section{A. pisum sugar porters are highly expressed at the gut interface}

Quantitative gene expression analysis of $A$. pisum sugar porter family transporters reveals that four transporters: 
ApST3, ACYPI004204; ApST4, ACYPI001980; ApST9, ACYPI001077; and ApST27, ACYPI009304, are highly expressed in adult females. Sugar porter family transporter expression is ranked ApST3 > ApST9 $>$ ApST4 $>A p S T 27$, in adult females, with the expression of all transporters above our $>10 \%$ GAPDH expression cut-off level (Figure 1A and B). Notably, three of these transporters (ApST3, ApST4, ApST9) are also the most highly expressed sugar porter transporters in A. pisum gut tissue. A fourth transporter ApST1, ACYPI001780, is expressed at low levels in whole insects $(<10 \% G A P D H)$, and is highly expressed $(>10 \%$ GAPDH) in A. pisum gut tissue (Figure 1C). Based on their differing expression profiles we can divide these four gut-expressed sugar porter family transporters into two groups; the first group includes ApST1, which has a strongly gut-bias expression pattern (up 33-fold in gut tissue relative to whole insect expression) (Figure 1D). The second group contains transporters ApST3, ApST4 and ApST9 that are universally highly expressed in whole adult female $A$. pisum and all tissues analyzed including embryos, heads and guts (Figure 1A). As well as being globally highly expressed, transporters ApST3 and ApST4 are enriched in gut tissue 2.3-fold and 5.9-fold, respectively, compared to whole insect expression levels (Figure 1C). Although highly expressed in all tissues analyzed, transporter ApST9 is not enriched in gut tissue, relative to whole insect expression levels (Figure 1C).

\section{A. pisum gut expressed sugar porters transport hexose sugars}

All four $A$. pisum gut expressed sugar transporters (ApST3 from our previous study [13] and ApST1, ApST4 and ApST9 reported here) were screened for hexose transport activity by functional complementation of the Saccharomyces cerevisiae hexose-uptake deficient strain EBY. VW4000 [16].

ApST3, a low affinity fructose/glucose transporter [13] was used as a positive control, and run alongside the functional screen of ApST1, ApST4 and ApST9. Yeast cells transformed with either ApST3 (positive control from [13]), ApST1, ApST4 or ApST9 expression constructs produced recombinant aphid transporter protein, which was detected in yeast total membrane fractions by anti-myc antibodies in western blots (Additional file 1: Figure S1). All aphid sugar transporters reported here (including the positive control ApST3) were detected below their calculated molecular weight when expressed in yeast (Additional file 1: Figure S1). The anomalous migration of transmembrane transporters on SDS-PAGE gels is attributed to the hydrophobic properties of the protein [17]. Immunoreactivity was not present in negative control cells that were transformed with an empty pDR195 expression construct (Additional file 1: Figure S1). Although all A. pisum transporters were expressed, only ApST3 (positive control from [13]) and

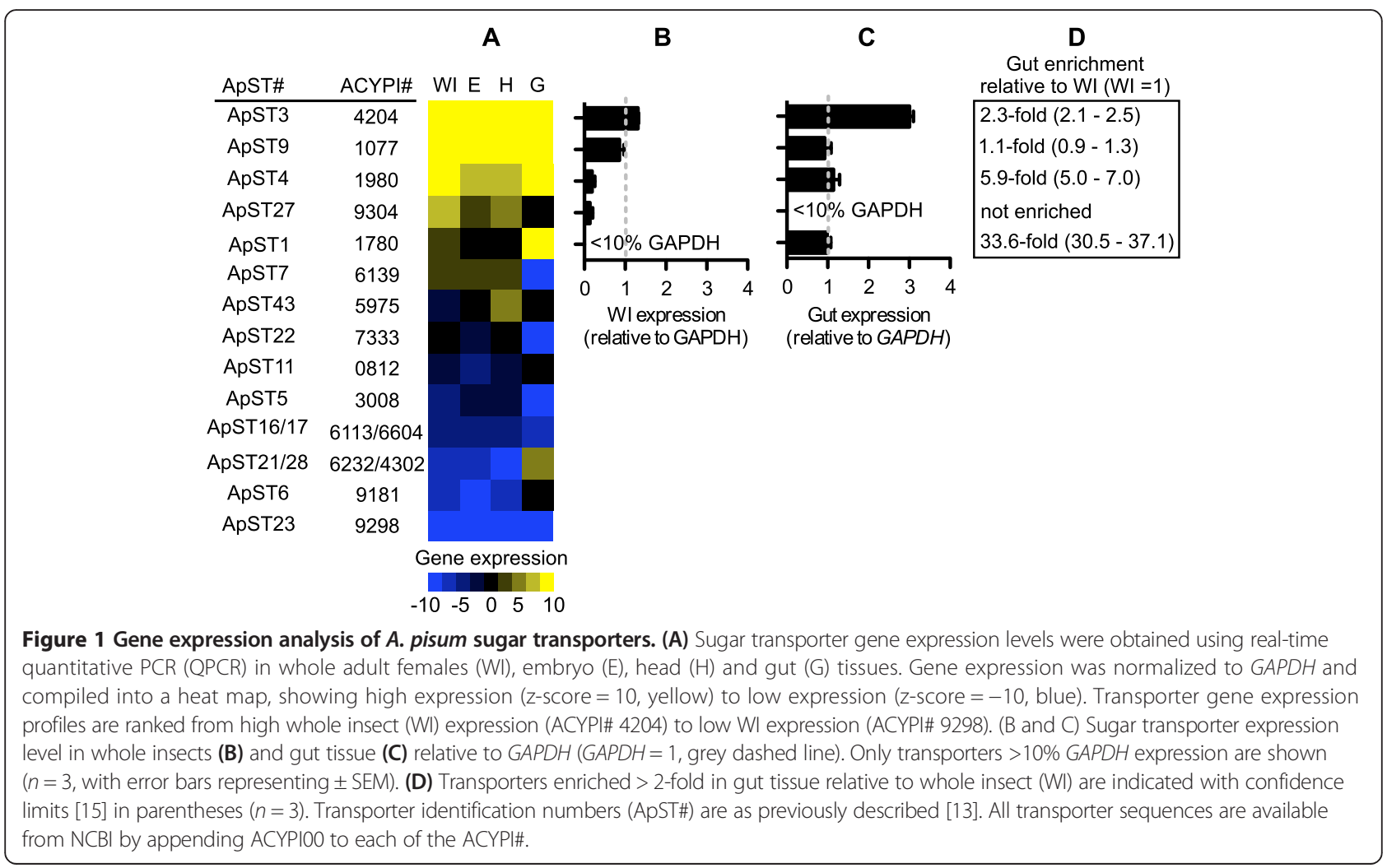


ApST4 were able to restore EBY.VW4000 growth on minimal media containing glucose, fructose, galactose and mannose as the sole carbon source (Figure 2). Importantly, negative control yeast cells (transformed with empty pDR195 expression vector) were unable to grow on minimal media plates containing these sugars (Figure 2). These results show that transporters ApST3 (as reported previously [13]) and ApST4 are functional hexose transporters that mediate the efficient transport of hexose sugars across the yeast plasma membrane.

\section{ApST4 is a facilitative hexose transporter}

ApST4 is a broad-spectrum hexose transporter. Substrate competition (inhibition) experiments with a 2-molar excess (100 mM initial extracellular concentration) of unlabeled competing sugar, demonstrate that glucose, mannose, fructose and galactose significantly reduce ${ }^{14} \mathrm{C}$ fructose uptake into yeast cells expressing ApST4 $(P<0.001$, one-way ANOVA followed by Dunnett's posttest, Figure 3A). ApST4 efficiently binds glucose $>$ mannose $>$ fructose, all reducing ${ }^{14} \mathrm{C}$-fructose uptake by $>50 \%$ compared to control uptake with no competing sugar. Galactose also competes with fructose uptake, but less efficiently, only reducing ${ }^{14} \mathrm{C}$-fructose uptake by $\sim 21 \%$ compared to control uptake with no competing sugar (Figure 3A). Competition assays with alcohol sugars: myo-inositol, sorbitol and mannitol revealed these do not compete with ${ }^{14} \mathrm{C}$-fructose uptake, and are therefore not recognized substrates (Figure $3 \mathrm{~A}$ ). In summary, functional complementation assays (Figure 2) and competition (inhibition) assays (Figure 3A) are consistent with ApST4 functioning as a broad-spectrum hexose transporter.

Major facilitator superfamily (MFS) transporters function as either facilitative transporters or secondary active transporters. Facilitative transporters (uniporters) mediate passive (equilibrative) solute transport across membranes,

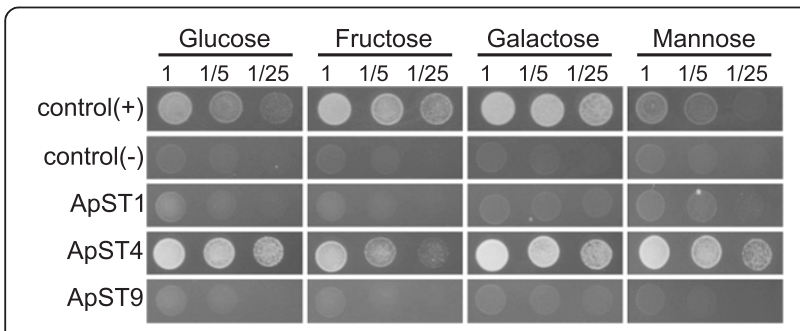

Figure 2 Functional identification of $A$. pisum glucose and fructose transporters. Saccharomyces cerevisiae hexose transport mutant EBY.W4000 was transformed with A. pisum gut expressed sugar transporters ApST1, ApST4 and ApST9. Positive control cells (+) were transformed with ApST3 [13] and negative control (-) cells were transformed with empty expression vector. Yeast cell suspensions containing $10 \mu \mathrm{l}$ of $1,1 / 5$ and $1 / 25 \mathrm{OD}_{600}$ units were plated on minimal media containing $60 \mathrm{mM}$ sugars (as indicated) as the sole carbon source. Recovery of mutant yeast growth was observed after 3 -days at $30^{\circ} \mathrm{C}$. moving solutes along their concentration gradients with no energy expenditure. In contrast, secondary active transporters (symporters) couple solute transport with monovalent cation (either $\mathrm{H}^{+}$or $\mathrm{Na}^{+}$) transport. Symporters require an electrochemical membrane potential to drive transport, allowing solutes to accumulate against their concentration gradient $[10,18,19]$. ApST4 transports glucose over a wide extracellular $\mathrm{pH}$ range, between $\mathrm{pH} 4.0$ and 9.0 (Figure 3B) and pre-treatment of yeast cells expressing ApST4 with proton ionophores carbonyl cyanide 3-chlorophenylhydrazone (CCCP) and 2,4-Dinitrophenol (DNP) does not significantly reduce glucose transport compared to untreated control cells (Figure 3B). Furthermore, there was no significant effect on ApST4 transport activity when $\mathrm{NaCl}$ in the transport buffer was substituted with choline chloride (Figure 3C). Our data demonstrates that ApST4 is a facilitated hexose transporter (uniporter) that functions independently of the electrochemical membrane potential.

Glucose and fructose uptake by ApST4 is concentration dependent, with transport rates saturating at high substrate concentrations, yielding an estimated $K_{\mathrm{m}}$ of $4.9 \pm 0.3 \mathrm{mM}$ for glucose and $31.0 \pm 2.6 \mathrm{mM}$ for fructose (Figure $4 \mathrm{~A}$ and $\mathrm{B}$ ).

\section{ApST4 shares sequence features with mammalian GLUT facilitative transporters}

ApST4 (full-length gene: $2066 \mathrm{bp}$ ) was reconstructed from sequence reads FF306288 and FF306499, present in the $A$. pisum full-length cDNA resource [20]. ApST4 comprises of a 403 bp 5' UTR, a 1476 bp coding sequence and a 185 bp 3' UTR that contains a putative polyadenylation signal (UAUAAA) 22 bp upstream from the poly-A tail. ApST4 contains 9 exons that map to a $40.8 \mathrm{kbp}$ region of scaffold 735 (total size $263.6 \mathrm{kbp}$ ) from $A$. pisum genomic scaffolds of assembly Acyr 2.0. Expression data retrieved from NCBIs dbEST, shows that ApST4 is expressed in all $A$. pisum sexual morphs including males (10 ESTs), sexual oviparous females (6 ESTs) and asexual females (7 ESTs). Partial sequence orthologs of ApST4 are present in the green peach aphid, Myzus persicae (3 ESTs); cotton aphid, Aphis gossypii (2 ESTs) and brown citrus aphid, Toxoptera citricida (1 EST).

The ApST4 protein encoded by the longest open reading frame (ORF) comprises 491 amino acids, with a predicted molecular weight of $53.4 \mathrm{kDa}$. ApST4 belongs to major facilitator superfamily (MFS), sugar porter (SP) family (T.C \# 2.A.1.1) and has 12 predicted transmembrane $\alpha-$ helices (as predicted by TMHMM, http://www.cbs.dtu. $\mathrm{dk} /$ services/TMHMM/), typical of related mammalian and insect GLUT transporters (Figure 5A). ApST4 shares $36 \%-45 \%$ sequence identity, and 50\% - 58\% similarity, with human GLUT class I transporters (GLUTs 1 - 4), with strong sequence conservation in the transmembrane 

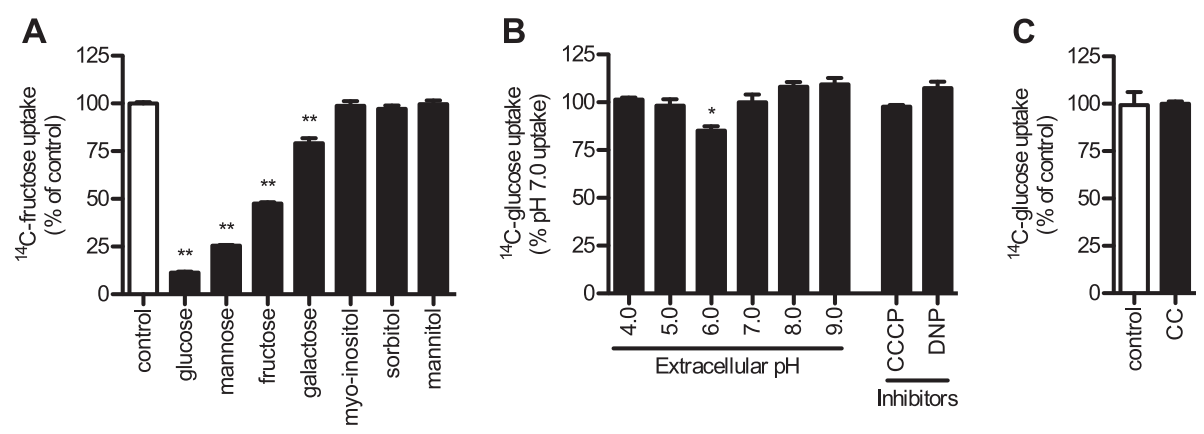

Figure 3 Functional characterization of ApST4 in whole yeast cells. Transport specificity of ApST4 was investigated by functional expression in EBY.WW4000 yeast cells (EBY-ST4 cells). (A) Inhibition of ${ }^{14} \mathrm{C}$-fructose uptake (50 mM initial extracellular concentration) in competition assays containing $100 \mathrm{mM}$ unlabeled sugars (as indicated), uptake is displayed as percentage of no competing sugar (control). (B) pH dependence of ${ }^{14} \mathrm{C}$-glucose uptake in EBY-ST4 cells using indicated extracellular pH; and in the presence of transport inhibitors: $1 \mu \mathrm{M}$ CCCP (Carbonyl cyanide 3-chlorophenylhydrazone) and $1 \mu \mathrm{M}$ DNP (2,4-Dinitrophenol). All uptake is displayed as percentage pH 7.0 transport. (C) $\mathrm{Na}^{+}$dependence of ${ }^{14} \mathrm{C}$-glucose uptake in EBY-ST4 cells using standard uptake buffer with addition of $50 \mathrm{mM} \mathrm{NaCl}$ (control), or in similar buffer were $\mathrm{NaCl}$ is replaced with an equimolar amount of choline chloride (CC). All uptake experiments were performed at $30^{\circ} \mathrm{C}$ and cells were collected by rapid-filtration after $10 \mathrm{~min}$. Sugar uptake was determined by liquid scintillation counting. Transport was corrected for background uptake into control (-) cells transformed with an empty expression vector. Each value is the mean $\pm \mathrm{SEM}, n=3$. Bars marked with asterisks are significantly different from controls. ${ }^{*} P<0.01$ and ${ }^{* *} P<0.001$; one way-ANOVA followed by Dunnett's post-test.

helix regions (Additional file 1: Figure S2). Both ApST3 [13] and ApST4 (reported here) contain signature sequences that are conserved across GLUT family members (Figure 5A and $\mathrm{B}$ ). Conserved residues in transmembrane helix 7 (TM7) that are critical for substrate binding [9,21] are consistent with ApST3 and ApST4 functioning as glucose and fructose transporters (Figure 5B).

\section{ApST4 is phylogenetically related to mammalian GLUT transporters}

The phylogenetic position of ApST4 was investigated by comparison with related transporters from humans and other insects. Insect taxa, including $N$. lugens, $T$. castaneum, A. melifera, D. melanogaster, P. humanus were chosen to represent the insect orders: Hemiptera,
Coleoptera, Hymenoptera, Diptera, and Phthiraptera, respectively. Phylogenetic reconstruction places APST4 in a well-supported clade containing unknown function insect transporters; mammalian GLUT class I transporters (GLUTs 1-4 and 14); and GLUT class II transporters (GLUTs 5, 7, 9, 11) (Figure 6) [22]. ApST4 (ACYPI001980) is most closely related to single orthologous insect transporters, all of unknown function, from $P$. humanus (PHUM247290), A. mellifera (LOC409424), and multiple (one to many) orthologs in T. castaneum (TC013486, TC014313 and TC013487) and N. lugens (AB550003 and AB549996). ApST4 and closely related (as yet uncharacterized) insect transporters are more distantly related to well-characterized mammalian GLUT class I facilitative hexose transporters (Figure 6) [22].
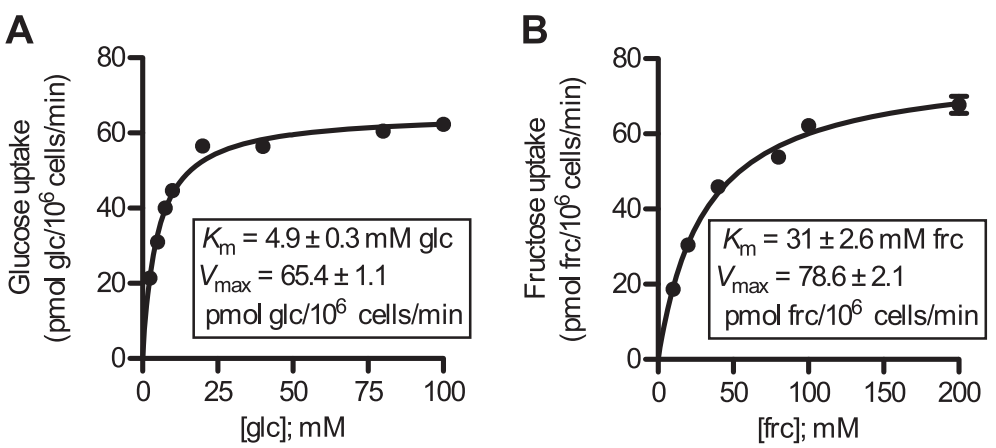

Figure 4 Glucose and fructose uptake kinetics of ApST4. Yeast cells expressing ApST4 were incubated in various concentrations of ${ }^{14} \mathrm{C}$-glucose and ${ }^{14} \mathrm{C}$-fructose (as indicated). (A) Glucose and (B) fructose uptake kinetics were fitted to Michaelis-Menten equations and $K_{\mathrm{m}}$ and $V_{\max }$ was determined by nonlinear regression using Prism 5.0c software. All uptake experiments were performed at $30^{\circ} \mathrm{C}$ and cells were collected by rapid-filtration after $10 \mathrm{~min}$. Sugar uptake was determined by liquid scintillation counting. Transport was corrected for background uptake into control (-) cells transformed with an empty expression vector. Each value is the mean $\pm \mathrm{SEM}, n=3$. 


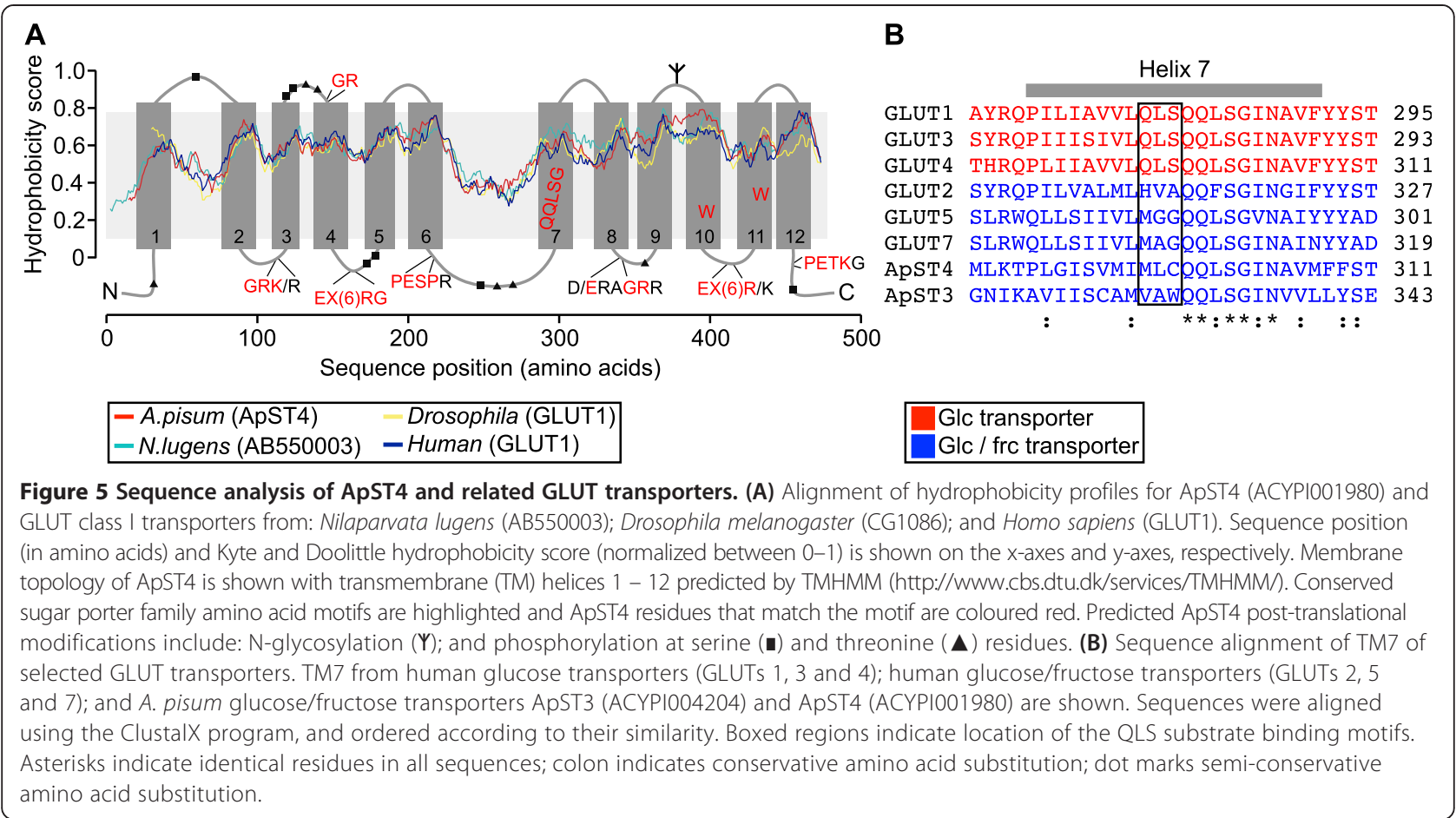

\section{Discussion}

Here, in an update to our original sugar transporter gene annotation [13], we have used a de novo search to identify 19 sugar porter family transporters in the latest version of the $A$. pisum genome (assembly Acyr_2.0). Compared to our previous study, which identified 29 putative sugar transporters [13], we present a subset of the original gene list (19/29 transporters) that match the TIGRFAMS sugar porter family motif (TIGR00879). The refinement of the original gene set is due in part, to a more stringent cut-off value used for transporter identification, combined with five gene models being withdrawn from the latest $A$. pisum genome assembly. The withdrawn gene models were previously identified as: ApST10, ACYPI007611; ApST33, ACYPI007211; ApST41, ACYPI006926; ApST51, ACYPI003842; and ApST55, NW_001921974 [13]. A reduced number of putative sugar transporter genes were also identified in de novo searches of other insect genomes (Additional file 1: Table S2). This reduction is again due to use of a more stringent cut-off used for whole genome de novo searches combined with use of updated genome assemblies.

Of the 19 annotated A. pisum sugar porter family transporters (matching the TIGRFAMs sugar porter family motif TIGR00879), four transporters: ApST1, ApST3, ApST4 and ApST9 are highly expressed and/or enriched in gut tissue. In our functional assays of these four transporters, only ApST3 (as reported previously [13]) and ApST4 (reported here) transport glucose and fructose. Our data is consistent with A. pisum using facilitative transporters ApST3 and ApST4 at the gut interface for import of glucose and fructose [3,5]. Based on transport function, we hypothesize that glucose and fructose moving passively from high concentrations in the gut lumen, to low concentrations of glucose and fructose in the haemolymph [24]. The functional role of ApST1 and ApST9 at the A. pisum gut interface remains unknown. Although ApST1 and ApST9 were expressed in the yeast hexose-transport deficient mutant EBY.VW4000 (Additional file 1: Figure S1), they both failed to functionally complement the yeast mutant (Figure 2). It is possible that ApST1 and ApST9 were not efficiently targeted to the yeast plasma membrane and were not available for sugar uptake. Alternatively, the hexose sugars used in our functional assays were not recognized substrates. Current work is underway to functionally express ApST1 and ApST9 and further investigate their transport properties and substrate selectivity.

Aphid transporters ApST3 and ApST4 are structurally, functionally and phylogenetically related to mammalian GLUTs. Both ApST3 and ApST4, like their human GLUT orthologs have a conserved sequence motif in transmembrane helix 7 (TM7) that is critical for substrate selectivity $[9,21,25]$ (Figure 5 and Additional file 1: Figure S3). Specifically, human GLUTs 1, 3 and 4, which transport glucose but not fructose have a conserved QLS amino acid motif in TM7; whereas GLUTs 2, 5, 7, 9 and 11 which transport glucose and fructose have amino acid residues: HVA, MGG, MAG, MAC, or GSA respectively, in the equivalent position $[9,21,25]$ (Figure 5B 


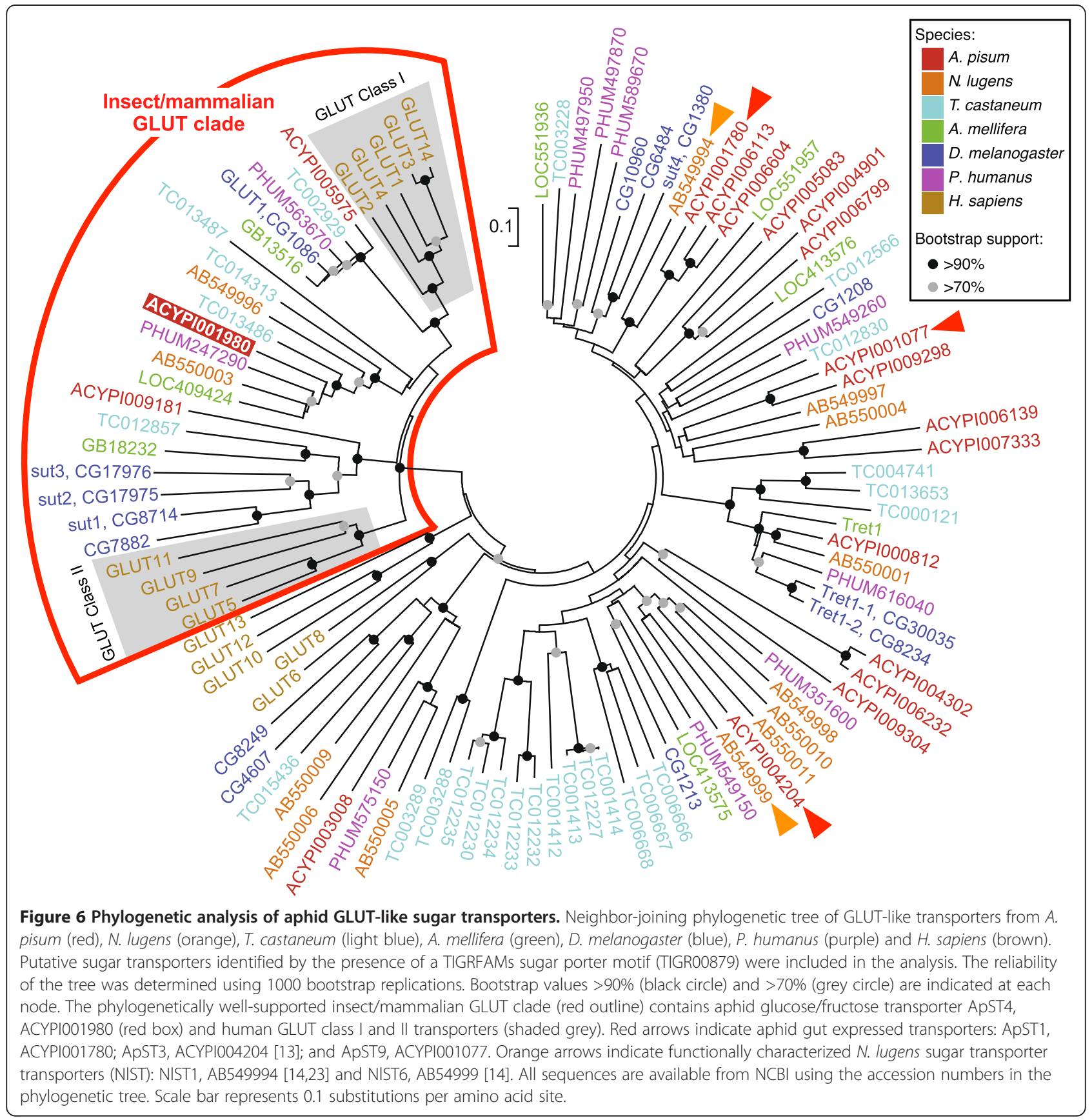

and Additional file 1: Figure S3). The functional role of the QLS motif for determining glucose specificity has been experimentally validated in two independent experiments [21,25]. First, a GLUT3/2 chimera, where the beginning of TM7 to the C-terminus of GLUT3, was replaced by the corresponding region of GLUT2, converted the protein to a glucose/fructose transporter, with GLUT2 kinetics [25]. Second, a substitution experiment, where GLUT2 had amino acids HVA replaced with QLS, and GLUT3 had amino acid QLS replaced with HVA resulted in partial reversal of their substrate specificities and uptake kinetics [21]. Insect GLUT-like transporters ApST3 [13], ApST4 (reported here) and NIST6 [14] are all facilitative glucose/fructose transporters. Sequence analysis, demonstrates that these transporters, similar to human GLUT glucose/fructose transporters, do not have a QLS-motif in TM7, consistent with glucose and fructose transport (Figure 5B and Additional file 1: Figure S3). In an extension of this observation, based on the presence of the QLS amino acid motif within TM7 for all insect GLUT class I orthologs included in our analysis (A. pisum, ACYPI005975; T. castaneum, TC002929; P. humanus, 
PHUM563670; D. melanogaster, CG1086 and A. mellifera, GB13516) (Additional file 1: Figure S3), we make the prediction that they are all specific for glucose (and not fructose). Current work is underway to further investigate structure/function relationships across insect GLUT class I orthologs.

Our functional screen specifically targeted sugar transporters belonging to the major facilitator superfamily, the largest family of characterized sugar transporters. However, it is possible that aphid transporters belonging to the solute:sodium symporter (SSS) family [transporter classification number (T.C \#) 2.A.21] also transport sugars. As with the sugar porter family, previously described, sugar transporters within the solute:sodium symporter family are also most extensively characterized in humans (Reviewed in [26]). Human sodium-glucose transporters, also known as $\mathrm{Na}^{+}$/glucose cotransporters (gene symbol SLC5A, protein symbol SGLT), mediate active transport of glucose i.e. they can move glucose against its concentration gradient. The $A$. pisum genome contains 7 solute:sodium symporter family transporters (Pfam sodium:solute symporter motif, PF00474), 6 of which are predicted to function as sodium/monocarboxylate cotransporters, and the other as a sodium/choline cotransporter (Additional file 1: Table S3). Importantly, our whole genome de novo searches did not identify $A$. pisum solute:sodium symporter family glucose transporters. Therefore, our data predicts that $A$. pisum uses only facilitative sugar transporters for sugar homeostasis, and not as in mammals, a combination of facilitate transporters and active transporters [10,11,26]. More generally, we hypothesize that aphid sugar homeostasis is energy-independent and maintained by facilitated diffusion (downhill movement) of sugars. This prediction necessitates a large family of related sugar transporters, with differing tissue expression patterns, and importantly subtle differences in substrate affinity between transporters.

\section{Conclusions}

In summary, this study demonstrates that $A$. pisum encodes a large family of sugar porter family transporters. Based on gene expression patters, only 4 transporters (ApST1, ApST3, ApST4 and ApST9) are predicted to play a functional role at the gut interface. Here we characterize A. pisum sugar transporter 4, ApST4, as a facilitative glucose/fructose transporter. Our gene expression and functional transport analyses are consistent with ApST4 playing a role in aphid sugar transport, functioning at the gut interface to transport glucose and fructose. Here, we demonstrate that ApST4 is, structurally, functionally and phylogenetically related to mammalian GLUT class I facilitative transporters. By placing transporters in a phylogenetic framework this work will facilitate prediction and testing of as yet uncharacterized sugar transporters across related taxa.

\section{Methods}

\section{Aphid culture}

Parthenogenetic females of the pea aphid, Acyrthosiphon pisum (Harris), lineage UY2 [27] were maintained on pre-flowering Vicia faba cv. The Sutton at $20^{\circ} \mathrm{C}$ in a long day regime of $16 \mathrm{~h}$ of light and $8 \mathrm{~h}$ of dark.

\section{De novo identification of sugar transporters}

The current version of the A. pisum genome Acyr_2.0 (GenBank assembly ID: GCA_000142985.2) contains 16932 gene models and 17718 protein models. Putative A. pisum sugar transporters matching the TIGRFAMs sugar porter (SP) family motif (TIGR00879 [28]) were identified in the latest $A$. pisum reference protein dataset using the hmmsearch program, which is part of the HMMER package (version 3.0) [29]. All identified sugar porter family transporters had a hmmsearch sequence score $>237.80$ (trusted cutoff).

For comparison, sugar porter family transporters were identified in a partial reference protein datasets from Nilaparvata lugens [30,31]; the reference protein datasets derived from whole genome sequence of $H$. sapiens; and whole genome sequences of 6 insect species. The insect species included in our comparative analysis were: Anopheles gambiae, Apis mellifera, Drosophila melanogaster, Nasonia vitripennis, Pediculus humanus, and Tribolium castaneum. Protein reference sequences used in the analysis are available from Ensembl Genomes (http:// ensemblgenomes.org/) and the version of the genome assembly used for the HMMER searches is listed in Additional file 1: Table S2. All identified sugar porter family transporters had a hmmsearch sequence score $>237.80$ (trusted cutoff). Following de novo identification of sugar transporters, the phylome database (phylomeDB, http:// phylomedb.org/), which is a complete phylogenetic analysis whole genomes [32] was searched for any additional homologs.

\section{Real-time quantitative PCR}

Real-time quantitative PCR (QPCR) was used to compare the gene expression profile of $16 \mathrm{~A}$. pisum sugar porter family transporters in different tissues using $2^{-\Delta \Delta C \mathrm{~T}}$ methodology [15]. All identified sugar porter family transporters were included, except ApST24, ApST25 and ApST26, which were excluded from the analysis due to the absence of supporting ESTs in whole adult female $A$. pisum. Primers were designed using Primer express software for real-time PCR v3.0 (Applied Biosystems) and specificity was checked using Primer-Blast (available at NCBI) against the $A$. pisum Refseq gene models and Acyr_2.0. Primer sequences are shown in Additional file 1: 
Table S4. For comparative gene expression analysis QPCR primers had amplification efficiencies $>90 \%$ and $<105 \%$. All experiments were performed in triplicate including no template controls and no reverse-transcription controls. Each reaction comprised of $1 \mathrm{X}$ Power SYBR green master mix (Applied Biosystems), $200 \mathrm{nM}$ of forward and reverse primers and cDNA derived from 8 ng total RNA from whole insect, head, gut and embryos. Sugar transporter gene expression was normalized to glyceraldehyde-3-dehydrogenase (GAPDH, ACYPI009769) in each tissue. In A. pisum we have established GAPDH as a reliable housekeeping gene to normalize gene expression as it is uniformly expressed throughout aphid development and across aphid tissues [13,33,34]. Normalized aphid transporter gene expression was and compiled into a heat map $(\mathrm{z}$-score $\times 10,[\mathrm{z}=($ each value - mean $) /$ standard deviation]). Yellow: $\mathrm{z}>0$ (high expression), blue $\mathrm{z}<0$ (low expression) and black $\mathrm{z}=0$.

\section{ApST expression constructs and yeast transformation}

Full-length coding sequences for gut expressed sugar transporters ApST1, ApST4, and ApST9 were amplified from whole adult female $A$. pisum cDNA using Phusion proof-reading polymerase (Finnzymes). All PCR primers contained a 5' optimized Kozak initiation sequence for efficient translation in yeast [35] and either a $5^{\prime}$ XhoI or EcoRV site and a 3' NotI site (primer sequences are shown in Additional file 1: Table S5). Amplified coding sequences of ApST1 and ApST4 were digested with XhoI and NotI and ApST9 was digested with EcoRV and NotI and cloned into the respective sites of the yeast shuttle vector pDR195, previously modified to encode a 3' c-Myc epitope [13]. The ApST3 yeast expression construct, with a 3' c-Myc epitope, was constructed previously, as described by [13]. ApST expression constructs were fully sequenced and used to transform Saccharomyces cerevisiae hexose transporter deletion mutant EBY.VW4000 [16] by the lithium acetate/PEG method [36]. Due to deletion of at least 20 hexose transporters, EBY.VW4000 has very low hexose transport activity, and is unable to grow on minimal media plates containing hexose sugars as the sole carbon source [16]. Transformants were selected on synthetic complete (SC) media, pH $5.6(0.17 \%$ yeast nitrogen base, $2 \%$ maltose, $1 \%$ agar supplemented with uracil drop-out mix) at $30^{\circ} \mathrm{C}$ for $3-4$ days. Recombinant transporter expression was detected by screening total membrane proteins from transformed cells by western blot using anti-c-Myc antibodies, as described in [13]. Positive transformants were replica plated on SC media lacking maltose but containing $60 \mathrm{mM}$ glucose, fructose, galactose or mannose and recovery of EBY.W4000 growth was assessed after 3 days at $30^{\circ} \mathrm{C}$. Controls were run in parallel and consisted of cells transformed with either the ApST3 fructose/glucose transporter [13] (positive control), or empty pDR195 vector (negative control).

\section{ApST uptake assays in whole yeast cells}

Uptake assays in whole yeast cells were based on methods described by [37]. Briefly, yeast cells were grown overnight at $30^{\circ} \mathrm{C}$ in liquid SC media containing $2 \%$ maltose, cells were harvested and washed twice in ice-cold transport buffer $\left(50 \mathrm{mM} \mathrm{NaPO}_{4}\right.$ buffer $\mathrm{pH} 7.0$ ) and resuspended to $30 \mathrm{OD}_{600} / \mathrm{ml}$ in the same buffer (unless indicated differently). For standard uptake assays, cells were incubated in a rotary shaker at $30^{\circ} \mathrm{C}$ and uptake assays were initiated by adding defined hexose sugars (50 mM initial extracellular concentration) labeled with D-[U- $\left.{ }^{14} \mathrm{C}\right]$-glucose (Perkin-Elmer) or D-[U- $\left.{ }^{14} \mathrm{C}\right]$ fructose (Perkin-Elmer), depending on the experiment. At given intervals 2 OD of cells were collected by rapid filtration onto nitrocellulose membranes $(0.2 \mu \mathrm{m}$ pore size) under vacuum and washed with an excess of ice-cold distilled water. Transport of ${ }^{14} \mathrm{C}$-glucose or ${ }^{14} \mathrm{C}$-fructose into yeast cells was determined by liquid scintillation counting. Glucose and fructose transport into yeast cells expressing ApST4 increased linearly for at least $30 \mathrm{~min}$. Thus, all uptake assays were stopped after $10 \mathrm{~min}$ in the linear phase of uptake. Sugar selectivity experiments were performed by adding a 2-fold molar excess of unlabeled sugar (100 mM initial extracellular concentration) to the standard ${ }^{14} \mathrm{C}$-labeled fructose uptake assay. $\mathrm{pH}$ dependence was analyzed by performing uptake assays in either $50 \mathrm{mM}$ citrate buffer ( $\mathrm{pH} 4-6)$, or $50 \mathrm{mM}$ phosphate buffer $(\mathrm{pH} 7-9)$, as indicated. The influence of $\mathrm{pH}$ gradient was determined by preincubating cells for $5 \mathrm{~min}$ with either $1 \mu \mathrm{M}$ 2,4-dinitrophenol (DNP) or $1 \mu \mathrm{M}$ carbonyl cyanide 3-chlorophenylhydrazone (CCCP). Sodium ion $\left(\mathrm{Na}^{+}\right)$dependence was analyzed by performing standard uptake assays with the addition of $50 \mathrm{mM} \mathrm{NaCl}$ to the transport buffer, control experiments were run in parallel, where $\mathrm{NaCl}$ was replaced with an equimolar amount of choline chloride. Kinetic parameters $K_{\mathrm{m}}$ and $V_{\text {max }}$ were estimated by fitting uptake rates at various substrate concentrations (as indicated) to the Michaelis-Menten equation using non-linear regression (Prism vs. 5.0c software, GraphPad). All uptake assays were performed in triplicate, using an independent yeast clone for each replicate and uptake data were corrected for background uptake into cells transformed with an empty pDR195 vector.

\section{Sugar transporter sequence analysis and phylogenetic analysis}

Sequence features in sugar transporter proteins were predicted using online search engines. Specifically, transmembrane $\alpha$-helix regions and membrane topology was predicted with the transmembrane using hidden Markov 
models (TMHMM) server (available at http://www.cbs. dtu.dk/services/TMHMM/) [38], or alternatively using Kyte and Doolittle hydrophobicity analysis (available at http://web.expasy.org/protscale/) [39]. Serine/threonine phosphorylation sites were predicted using the NetPhos 2.0 server (available at http://www.cbs.dtu.dk/services/ NetPhos/) [40]. Transporter N-linked glycosylation sites were predicted using NetNGlyc 1.0 server (available at http://www.cbs.dtu.dk/services/NetNGlyc/).

For phylogenetic analysis, protein sequences were aligned using MUSCLE [41] and ambiguously aligned positions were excluded by trimAL v1.3 [42]. Neighbour joining (NJ) phylogenetic trees, with 1000 bootstrap replicates, were constructed using MEGA5.2.2 [43].

\section{Availability of supporting data}

All data for the phylogenetic analysis (presented in Figure 6), which includes: sugar porter (SP) family protein sequences; MUSCLE aligned protein sequences; and the neighbor-joining phylogenetic tree are publically available in the LabArchives repository [22].

\section{Additional file}

Additional file 1: Figure S1. Western blot analysis of recombinant A. pisum sugar transporters expressed in yeast. Figure S2. Sequence alignment and transmembrane predictions for human GLUT class I transporters (GLUTs 1 - 4) and related insect transporters from: Acyrthosiphon pisum, Drosophila melanogaster and Nilaparvata lugens. Figure S3. Sequence alignment of transmembrane 7 (TM7) for human GLUT class I transporters (GLUTs 1-4 and 14), class II transporters (GLUTs 5, 7, 9 and 11) and insect orthologs. Table S1. Summary of annotated A. pisum sugar porter family transporters. Table S2. de novo identification of sugar porter (SP) family transporters [transporter classification number (T.C \#) 2.A.1.1] in insect genomes. Table S3. Summary of annotated A. pisum solute:sodium symporter (SSS) family transporters. Table S4. Quantitative PCR primers for A. pisum sugar transporters and housekeeping gene glyceraldehyde-3phosphate dehydrogenase (GAPDH). Table S5. A. pisum sugar transporter coding sequence primers for Saccharomyces cerevisiae expression constructs.

\section{Competing interests}

The authors declare that they have no competing interests.

\section{Authors' contributions}

DRGP and JAG conceived and designed the experiments. DRGP performed the experiments. DRGP and JAG analyzed the data. DRGP wrote the manuscript. Both authors read and approved the final manuscript.

\section{Acknowledgements}

We thank Alex C. C. Wilson (University of Miami) and Angela E. Douglas (Cornell University) for useful discussion and critical reading of the manuscript; and three anonymous reviewers whose comments helped improve the final manuscript. We acknowledge the generosity of Eckhard Boles (Goethe University Frankfurt am Main) for the contribution of S. cerevisiae strain EBY.WW4000. This work was supported by Biotechnology and Biological Sciences Research Council Grant BB/E006280/1 (to JAG.).

Received: 20 March 2014 Accepted: 13 June 2014

Published: 4 August 2014

\section{References}

1. Febvay G, Rahbe Y, Rynkiewicz M, Guillaud J, Bonnot G: Fate of dietary sucrose and neosynthesis of amino acids in the pea aphid, Acyrthosiphon pisum, reared on different diets. J Exp Biol 1999, 202(19):2639-2652

2. Rhodes JD, Croghan PC, Dixon AFG: Uptake, excretion and respiration of sucrose and amino acids by the pea aphid Acyrthosiphon pisum. J Exp Biol 1996, 199(6):1269-1276.

3. Rhodes JD, Croghan PC, Dixon AFG: Dietary sucrose and oligosaccharide synthesis in relation to osmoregulation in the pea aphid, Acyrthosiphon pisum. Physiol Entomol 1997, 22(4):373-379.

4. Price DRG, Karley AJ, Ashford DA, Isaacs HV, Pownall ME, Wilkinson HS, Gatehouse JA, Douglas AE: Molecular characterisation of a candidate gut sucrase in the pea aphid, Acyrthosiphon pisum. Insect Biochem Mol Biol 2007, 37(4):307-317.

5. Ashford DA, Smith WA, Douglas AE: Living on a high sugar diet: the fate of sucrose ingested by a phloem-feeding insect, the pea aphid Acyrthosiphon pisum. J Insect Physiol 2000, 46(3):335-341.

6. Cristofoletti PT, Ribeiro AF, Deraison C, Rahbe Y, Terra WR: Midgut adaptation and digestive enzyme distribution in a phloem feeding insect, the pea aphid Acyrthosiphon pisum. J Insect Physiol 2003, 49(1):11-24

7. Reddy VS, Shlykov MA, Castillo R, Sun El, Saier MH Jr: The major facilitator superfamily (MFS) revisited. FEBS J 2012, 279(11):2022-2035.

8. Thorens B, Mueckler M: Glucose transporters in the 21 st century. Am J Physiol Endocrinol Metab 2010, 298(2):E141-E145.

9. Manolescu AR, Witkowska K, Kinnaird A, Cessford T, Cheeseman C: Facilitated hexose transporters: new perspectives on form and function. Physiology 2007, 22:234-240.

10. Saier MH: Families of transmembrane sugar transport proteins. Mol Microbiol 2000, 35(4):699-710.

11. Mueckler M, Thorens B: The SLC2 (GLUT) family of membrane transporters. Mol Asp Med 2013, 34(2-3):121-138.

12. International Aphid Genomics Consortium: Genome sequence of the pea aphid Acyrthosiphon pisum. PLoS Biol 2010, 8(2):e1000313.

13. Price DRG, Tibbles K, Shigenobu S, Smertenko A, Russell CW, Douglas AE, Fitches E, Gatehouse AMR, Gatehouse JA: Sugar transporters of the major facilitator superfamily in aphids; from gene prediction to functional characterization. Insect Mol Biol 2010, 19:97-112.

14. Kikuta S, Kikawada T, Hagiwara-Komoda Y, Nakashima N, Noda H: Sugar transporter genes of the brown planthopper, Nilaparvata lugens: a facilitated glucose/fructose transporter. Insect Biochem Mol Biol 2010, 40(11):805-813.

15. Livak KJ, Schmittgen TD: Analysis of relative gene expression data using real-time quantitative PCR and the $2^{-\Delta \Delta C T}$ Method. Methods 2001, 25(4):402-408.

16. Wieczorke R, Krampe $S$, Weierstall $T$, Freidel $K$, Hollenberg CP, Boles E: Concurrent knock-out of at least 20 transporter genes is required to block uptake of hexoses in Saccharomyces cerevisiae. FEBS Lett 1999, 464(3):123-128.

17. Ward A, Sanderson NM, O'Reilly J, Rutherford NG, Poolman B, Henderson PJF: The amplified expression, identification, purification, assay, and properties ofhexahistidine-tagged bacterial membrane transport proteins. In Membrane Transport. Edited by Baldwin SA. Oxford: Oxford University Press; 2000:141-166.

18. Marger MD, Saier MH: A major superfamily of transmembrane facilitators that catalyze uniport, symport and antiport. Trends Biochem Sci 1993, 18(1):13-20

19. Saier $\mathrm{MH}$ : A functional-phylogenetic classification system for transmembrane solute transporters. Microbiol Mol Biol Rev 2000, 64(2):354-411.

20. Shigenobu S, Richards S, Cree A, Morioka M, Fukatsu T, Kudo T, Miyagishima S, Gibbs R, Stern D, Nakabachi A: A full-length cDNA resource for the pea aphid, Acyrthosiphon pisum. Insect Mol Biol 2010, 19(s2):23-31.

21. Seatter MJ, De la Rue SA, Porter LM, Gould GW: QLS motif in transmembrane helix VII of the glucose transporter family interacts with the C-1 position of D-glucose and is involved in substrate selection at the exofacial binding site. Biochemistry 1998, 37(5):1322-1326.

22. Labarchives Repository. http://dx.doi.org/10.6070/H48P5XG5.

23. Price DRG, Wilkinson HS, Gatehouse JA: Functional expression and characterisation of a gut facilitative glucose transporter, NIHT1, from the phloem-feeding insect Nilaparvata lugens (rice brown planthopper). Insect Biochem Mol Biol 2007, 37:1138-1148. 
24. Moriwaki N, Matsushita K, Nishina M, Kono Y: High concentrations of trehalose in aphid hemolymph. Appl Entomol Zool 2003, 38(2):241-248.

25. Arbuckle MI, Kane S, Porter LM, Seatter MJ, Gould GW: Structure-function analysis of liver-type (GLUT2) and brain-type (GLUT3) glucose transporters: expression of chimeric transporters in Xenopus oocytes suggests an important role for putative transmembrane helix 7 in determining substrate selectivity. Biochemistry 1996, 35(51):16519-16527.

26. Wright EM, Loo DDF, Hirayama BA: Biology of human sodium glucose transporters. Physiol Rev 2011, 91(2):733-794.

27. Darby AC, Douglas AE: Elucidation of the transmission patterns of an insect-borne bacterium. Appl Environ Microbiol 2003, 69(8):4403-4407.

28. Haft DH, Loftus BJ, Richardson DL, Yang F, Eisen JA, Paulsen IT, White O: TIGRFAMs: a protein family resource for the functional identification of proteins. Nucleic Acids Res 2001, 29(1):41-43.

29. Eddy SR: Profile hidden Markov models. Bioinformatics 1998, 14(9):755-763.

30. Noda H, Kawai S, Koizumi Y, Matsui K, Zhang Q, Furukawa S, Shimomura M, Mita K: Annotated ESTs from various tissues of the brown planthopper Nilaparvata lugens: a genomic resource for studying agricultural pests. BMC Genomics 2008, 9:117.

31. Bass C, Hebsgaard MB, Hughes J: Genomic resources for the brown planthopper, Nilaparvata lugens: transcriptome pyrosequencing and microarray design. Insect Sci 2012, 19(1):1-12.

32. Huerta-Cepas J, Capella-Gutierrez S, Pryszcz LP, Marcet-Houben M, Gabaldon T: PhylomeDB v4: zooming into the plurality of evolutionary histories of a genome. Nucleic Acids Res 2014, 42(Database issue):D897-902.

33. Price DR, Feng H, Baker JD, Bavan S, Luetje CW, Wilson AC: Aphid amino acid transporter regulates glutamine supply to intracellular bacterial symbionts. Proc Natl Acad Sci U S A 2014, 111(1):320-325.

34. Price DRG, Duncan RP, Shigenobu S, Wilson ACC: Genome expansion and differential expression of amino acid transporters at the aphid/Buchnera symbiotic interface. Mol Biol Evol 2011, 28(11):3113-3126.

35. Kozak M: Structural features in eukaryotic mRNAs that modulate the initiation of translation. J Biol Chem 1991, 266(30):19867-19870.

36. Gietz RD, Schiestl RH: Quick and easy yeast transformation using the LiAc/SS carrier DNA/PEG method. Nat Protoc 2007, 2(1):35-37.

37. Sauer N, Stolz J: Expression of foreign transport proteins in yeast. In Membrane Transport. Edited by Baldwin SA. Oxford: Oxford University Press; 2000:79-105.

38. Krogh A, Larsson B, von Heijne G, Sonnhammer EL: Predicting transmembrane protein topology with a hidden Markov model: application to complete genomes. J Mol Biol 2001, 305(3):567-580.

39. Kyte J, Doolittle RF: A simple method for displaying the hydropathic character of a protein. J Mol Biol 1982, 157(1):105-132.

40. Blom N, Gammeltoft S, Brunak S: Sequence and structure-based prediction of eukaryotic protein phosphorylation sites. J Mol Biol 1999, 294(5):1351-1362

41. Edgar RC: MUSCLE: multiple sequence alignment with high accuracy and high throughput. Nucleic Acids Res 2004, 32(5):1792-1797.

42. Capella-Gutierrez S, Silla-Martinez JM, Gabaldon T: trimAl: a tool for automated alignment trimming in large-scale phylogenetic analyses. Bioinformatics 2009, 25(15):1972-1973.

43. Tamura K, Peterson D, Peterson N, Stecher G, Nei M, Kumar S: MEGA5: molecular evolutionary genetics analysis using maximum likelihood, evolutionary distance, and maximum parsimony methods. Mol Biol Evol 2011, 28(10):2731-2739.

doi:10.1186/1471-2164-15-647

Cite this article as: Price and Gatehouse: Genome-wide annotation and functional identification of aphid GLUT-like sugar transporters. BMC Genomics 2014 15:647.

\section{Submit your next manuscript to BioMed Central and take full advantage of:}

- Convenient online submission

- Thorough peer review

- No space constraints or color figure charges

- Immediate publication on acceptance

- Inclusion in PubMed, CAS, Scopus and Google Scholar

- Research which is freely available for redistribution

Submit your manuscript at www.biomedcentral.com/submit
C Biomed Central 